\title{
1 Genetic perturbation enhances functional heterogeneity in alkaline
}

2 phosphatase

3

4 Morito Sakuma $^{\# 1,2}$, Shingo Honda ${ }^{1}$, Hiroshi Ueno ${ }^{1}$, Kentaro Miyazaki ${ }^{3,4}$, Nobuhiko

5 Tokuriki $^{2^{*}}$, and Hiroyuki Noji ${ }^{*}$

6

$7{ }^{1}$ Department of Applied Chemistry, Graduate School of Engineering, The University

8 of Tokyo, Tokyo, Japan

$9{ }^{2}$ Michael Smith Laboratories, The University of British Columbia, British Columbia,

10 Canada

$11{ }^{3}$ Bioproductuon Research Institute, National Institute of Advanced Industrial Science

12 and Technology, Ibaraki, Japan

$13{ }^{4}$ Department of Computational Biology and Medical Science, Graduate School of

14 Frontier Sciences, The University of Tokyo, Chiba, Japan

15

$16{ }^{\star}$ Correspondence: hnoji@g.ecc.u-tokyo.ac.jp (H.N.) or tokuriki@msl.ubc.ca (N.T.)

17

\# Present address: Michael Smith Laboratories, The University of British Columbia,

192185 East Mall, Vancouver, BC, V6T 1Z4, Canada

21 Keywords: enzyme function; single-molecule assay; functional heterogeneity; 


\section{Abstract}

25 Enzymes inherently exhibit molecule-to-molecule heterogeneity in catalytic activity or

26 function, which underlies the acquisition of new functions in evolutionary processes.

27 However, correlations between the functional heterogeneity of an enzyme and its

28 multi-functionality or promiscuity remain elusive. In addition, the modulation of

29 functional heterogeneity upon genetic perturbation is currently unexplored. Here, we

30 quantitatively analyzed functional heterogeneity in the wild-type and 69 single-point

31 mutants of Escherichia coli alkaline phosphatase (AP) by employing single-molecule

32 assay with a femtoliter reactor array device. Most mutant enzymes exhibited higher

33 functional heterogeneity than the wild-type enzyme, irrespective of catalytic activity.

34 These results indicated that the wild-type AP minimizes functional heterogeneity, and

35 single-point mutations can significantly expand the span of functional heterogeneity

36 in AP. Moreover, we identified a clear correlation between functional heterogeneity

37 and promiscuous activities. These findings suggest that enzymes can acquire

38 greater functional heterogeneity following marginal genetic perturbations that concomitantly promote catalytic promiscuity. 


\section{Introduction}

As our understanding of enzymes matured over the years, the once simple and prevailing "one enzyme, one function" paradigm has undergone a change.

44 Advances in technology and statistical methods have revealed that enzymes intrinsically exhibit molecule-to-molecule heterogeneity in catalytic activity or substrate specificity, even in a clonal population ${ }^{1-3}$. Such functional heterogeneity among molecules persists on time scales far beyond thermal fluctuations - from milliseconds to hours in some enzymes ${ }^{4-18}$. Therefore, it can be referred to as longlived functional substates; for simplicity of discussion in this work, we define

50 "functional substate" as the long-lived and distinct functional states among the enzyme molecules with the same genotype, independent of any genetic mutation. The functional substate of enzyme molecules is considered key for molecular

53 mechanisms involving enzymatic functions ${ }^{4-16}$ and may be driving the acquisition of

54 new functions during evolution ${ }^{17-20}$. Specifically, functional substates have been proposed to underlie enzyme promiscuity (or multi-functionality): within an ensemble

56 of molecules, certain dominant functional substates can exclusively perform the 57 native function, whereas some minor functional substates catalyze secondary or 58 promiscuous reactions ${ }^{3,21-24}$. Moreover, it is postulated that the evolutionary 
60 functional substates ${ }^{3,21-24}$. This view hypothesizes that enzymes with well-optimized

61 native activities have become specialists throughout evolution by converging toward

62 a limited number of functional substates. Conversely, it can be expected that random

63 mutations have the potential to disrupt the functional substates optimized for the

64 native activity, thus to enhance promiscuous functions that were previously

65 supported by a low substate population. Hence, manipulating the functional substate

66 of an enzyme could be key to understanding enzyme evolution and harnessing

67 promiscuous activities for enzyme engineering. The majority of our understanding regarding functional substates is based on

69 structural studies. Such long-lived functional substates are thought to originate from

70 stable conformational substates revealed by structural analyses using X-ray

71 crystallography, nuclear magnetic resonance (NMR), and molecular dynamics (MD)

72 simulations ${ }^{25,26}$. Previous studies have revealed that function-altering mutations can

73 perturb the protein structure and increase conformational heterogeneity ${ }^{27,28}$.

74 However, it is unclear whether such conformational heterogeneity results in

75 functional substates. Moreover, the averaging of a population of enzymes in

76 crystallography or NMR would mean subtle conformational changes in enzymes,

77 even in stable and distinct populations of functional substates, barely detectable by

78 the structural analysis and hence remain obscured ${ }^{29,30}$. Therefore, direct 
experimental measurement of functional substates that link each enzyme molecule

with its functional characteristics is essential to advance our understanding.

microreactor/microfluidic devices and microscopic measurements has revolutionized

83 the study of enzymatic reactions ${ }^{10-16,31-37}$. Recent studies, which utilized a femtoliter

84 reactor array device (FRAD) or similar technologies, statistically analyzed the

85 functional heterogeneity in an enzyme population performing thousands of single-

86 molecule reactions conducted in parallel ${ }^{14-16,34-37}$. The single-molecule assay can

87 thus provide significant information to determine the functional substate, such as a

88 number of activity states or the extent of the functional heterogeneity, i.e., width of

89 the distribution in catalytic activities. The existence of functional substates observed

90 over time scales of tens of minutes or hours was reported for $\beta$-galactosidase (GAL),

$91 \quad \beta$-glucuronidase (GUS), and alkaline phosphatase (AP) ${ }^{14-16,34,35}$. Those enzymes

92 show one or two distinct population in the functional heterogeneity. Interestingly,

93 wild-type GUS exhibits a relatively low heterogeneity (narrow distribution), whereas

94 the mutants of GUS having high catalytic activity toward promiscuous substrates

95 exhibit higher heterogeneity (broader distribution) than the wild-type ${ }^{34}$. However,

96 answers to many of the fundamental questions concerning functional substates

97 remain elusive. Has evolution enriched specific functional substates during the 
98 functional optimization of natural enzymes? How easily can mutations modulate

99 functional substates? Are functional substates of a particular native-like substrate

100 associated with promiscuous activity?

101 In this study, we employed single-molecule technology using the FRAD to

102 measure catalytic activity of the wild-type and 69 mutants of Escherichia coli (E.coli)

103 AP. We then analyzed their functional substates based on the functional

104 heterogeneity. We found that the wild-type AP (AP-wt) exhibited the lowest functional

105 heterogeneity, and most mutants presented greater functional heterogeneity than the

106 AP-wt. Hence, a single-point mutation in the AP-wt significantly expands its

107 functional substates. We also tested potential correlations involving functional

108 heterogeneity and promiscuity by measuring the catalytic activity of mutants

109 displaying different extent of functional heterogeneity against various promiscuous

110 substrates. Mutants exhibiting high functional heterogeneity also showed high

111 promiscuous activities, which suggests that the expansion of the functional substates

112 of native function could confer catalytic promiscuity. This study provides the first

113 quantitative evidence of the contribution of molecule-to-molecule heterogeneity for

114 enzyme promiscuity and its evolution. 


\section{Results}

\section{Single-molecule assay of E. coli AP} We employed E. coli AP as a model to characterize heterogeneity in catalytic

119 activities. AP is a homodimeric metalloenzyme encompassing two $\mathrm{Zn}^{2+}$ ions and one

$120 \mathrm{Mg}^{2+}$ in its active site (Fig. 1a). AP was expressed using the PURE system, a

121 reconstituted cell-free protein synthesis system optimized in our previous studies ${ }^{16,36}$.

122 The catalytic activity of AP expressed in the PURE system can be measured using a

123 FRAD without purification ${ }^{16}$. After AP molecules were expressed, the PURE reaction

124 solution was diluted and mixed with a fluorescein-based fluorogenic phosphate

125 monoesters substrate (fluorescein diphosphate, FDP) (Fig. 1a). Subsequently, to

126 measure single enzyme activity, the enzyme assay mixture was applied to a flow

127 channel of the FRAD displaying over 46,000 reactors (Fig. 1a and Supplementary

128 Fig.1). A fluorinated oil (FC-40) was then injected into the flow channel to displace

129 the enzyme assay mixture and seal the reactors (Supplementary Fig.1b), ensuring

130 negligible cross-reactor diffusion levels over the course of the experiment

131 (Supplementary Fig. 2). The single enzyme activity was measured by the increase

132 in the fluorescence intensity in the reactors (Fig. 1b). The single-molecule assay was

133 performed according to previously optimized reaction conditions using $1 \mathrm{mM}$ FDP, 
134 since AP exhibits catalytic activity even after $6 \mathrm{~h}$ without any significant decrease in

135 activity ${ }^{16}$.

137 Measurement of heterogeneity in catalytic activities

138 The catalytic activity and heterogeneity in catalytic activities among AP-wt

139 molecules were evaluated based on the distribution of fluorescence intensity

140 observed in all reactors (Fig. 1c). The histograms of fluorescence intensity typically

141 showed two main discrete peaks: a peak of low fluorescence intensity was derived

142 from enzyme-free reactors (peak 1) and another (peak 2) with higher fluorescence

143 intensity represents reactors containing a single enzyme molecule. We occasionally

144 observed an additional minor peak (peak 3) containing two or more enzyme

145 molecules, which exhibited approximately 2-fold higher intensity than peak $2^{16,35}$.

146 The enzyme solution was diluted in order to reduce the population of peak 3 reactors,

147 and if peak 3 remained, it was excluded from the analysis. We measured heterogeneity in catalytic activities by fitting the

149 distribution of peaks 1 and 2 with a Gaussian function to determine the mean

150 intensity $\left(M I_{1}\right.$ or 2$)$ and standard deviation $\left(S D_{1}\right.$ or 2$)$ of the peaks (see Methods).

151 Using the $M I$ of the two peaks and measurement time $(t)$, the mean catalytic activity

$152(M A)$ across all single enzyme molecules is defined as: 


$$
M A=\frac{M I_{2}-M I_{1}}{t}, \text { in a.u. } / \min
$$

154 The MA of the AP-wt with $1 \mathrm{mM}$ FDP was determined to be $13.2 \pm 0.8$ (a.u./min)

155 (Extended Data), which corresponds to the turnover rate of $19 \mathrm{~s}^{-1}$ calculated using

156 the calibration curve (Supplementary Fig. 4a), consistent with the turnover rate

157 determined in bulk measurement, $26 \mathrm{~s}^{-1}$ (Supplementary Fig. 3b and $\mathbf{4 b}$ ). The concentration of FDP in the single-molecule assay was sufficiently higher than the

$159 K_{\mathrm{m}}$ obtained in bulk measurements $\left(K_{\mathrm{m}}=8 \mu \mathrm{M}\right)$ (Supplementary Fig. 3a and b).

160 Heterogeneity in catalytic activities was defined as the coefficient of variation $\left(C V_{\mathrm{i}}=\right.$

$161 S D_{\mathrm{i}} / M l_{\mathrm{i}}, \mathrm{i}=1$ or 2$)$. The $C V$ of $\mathrm{AP}-\mathrm{wt}\left(C V_{2}: C V\right.$ for peak $2=12 \pm 3 \%$ in nine independent measurements) was approximately two-fold higher than the inherent noise of the system estimated from the $C V$ of peak $1\left(C V_{1}=6 \pm 1 \%\right)$ (see Methods).

164 Finally, we calculated the "functional heterogeneity" of AP-wt as the normalized CV $\left(C V_{n}\right)$ by compensating for system noise, $C V_{1}$, as:

$$
C V_{n}=\sqrt{C V_{2}^{2}-C V_{1}^{2}}
$$

The resulting $C V_{\mathrm{n}}$ for $\mathrm{AP}$-wt was $10 \pm 3 \%$. 
172 from a mutagenized library that we previously generated ${ }^{36}$. In addition, we prepared

173 four mutants (D101S, G118D, D153S, and K328R) that were identified as highly

174 active against FDP (see Methods). In total, 69 mutants were examined by single-

175 molecule assay with FRAD. The activity of each mutant was measured in duplicate,

176 and $M A$ (a.u./min) and functional heterogeneity $\left(C V_{\mathrm{n}}, \%\right)$ were analyzed. Variations

177 in the distribution were observed, as the single enzyme peak (peak 2) could

178 represent a single peak or comprise two neighboring peaks, for example, E66V and

179 D128V (Supplementary Fig. 5). A double-Gaussian function could not explicitly fit

180 the neighboring two peaks in some mutants, and thus, all distributions were fitted

181 with a single Gaussian function. The replicates for all mutants exhibited good

182 reproducibility in determining $M A$ and $C V_{\mathrm{n}}$ (Supplementary Fig. 6 and Extended

183 Data).

The four high-activity mutant variants, D101S, G118D, D153S, and K328R -

185 all located around the catalytic reaction center (within $10 \AA$ from the ligand) -

186 exhibited 4 to 13-fold higher activity than AP-wt (Fig. 2a). Most randomly chosen

187 mutations only marginally affected $M A$; almost all mutants presented an $M A$ within 2-

188 fold of the wild-type (Fig. 2a). Furthermore, the 65 randomly selected mutants did

189 not reveal any clear correlation between catalytic activity and the distance of the

190 mutated site from the catalytic reaction center (Fig. 2a and b). 
We found that the AP-wt exhibited a single narrow distribution peak

192 (Supplementary Fig. 5); its $C V_{\mathrm{n}}$ being the lowest among all mutants $\left(C V_{\mathrm{n}}=10 \%\right)$.

193 Most mutants showed significantly higher heterogeneity than the AP-wt. On average,

194 the $C V_{n}$ of mutants was 2-fold higher (20\%), and some mutants showed up to 3-fold

195 higher $C V_{\mathrm{n}}$ than the AP-wt. Intriguingly, there was no apparent correlation between

196 the $C V_{\mathrm{n}}$ and $M A$ (Fig. 2a). This observation suggests that functional heterogeneity is

197 principally independent of the level of catalytic activity and that the molecular

198 mechanism for gaining functional heterogeneity differs from that for catalysis

199 enhancement.

200 Across all mutants, we observed a broad range of combinations between

201 functional heterogeneity and catalytic activity (Fig. 2d-f and Supplementary Fig. 7).

202 Indeed, some mutants exhibited a single peak, yet displayed catalytic activities

203 different from the wild-type (Fig. 2d). We hypothesized that, in these specific cases,

204 the mutation likely modulates the transition states of the kinetic bottleneck steps

205 without disturbing the populations of functional substates. By contrast, in other

206 mutants, one population exhibited catalytic activity comparable to that of the AP-wt

207 (Fig. 2e), and an additional population was also found, resulting in a bimodal

208 distribution. Mutations that lead to shifts between sub-populations, visible through

209 the activity change in the ensemble average of enzymes, can underlie the 
210 emergence of a new functional substate within a clonal population. Such mutations

211 may act to stabilize functional substates, which were minor in the AP-wt. Finally,

212 another set of mutations resulted in two peaks, although both peaks were shifted

213 from that of the AP-wt population (Fig. 2f), suggesting that these mutations can

214 simultaneously modulate enzyme kinetics and the population balance between

215 various functional substates. Taken together, these observations suggest that a

216 mutation can easily alter the balance of AP functional substates, and the effect on

217 the population balance differs widely, depending on the specific mutation.

219 exhibited distinct differences in terms of heterogeneity in catalytic activities (Fig. 3).

220 D101S, D153S, and K328R exhibited shifted and broader distributions than the AP-

221 wt. Especially, D153S showed the highest $C V_{n}$ among the mutants. However,

222 G118D did not enhance heterogeneity despite having four-fold higher catalytic

223 activity than the AP-wt, i.e., the $C V_{n}$ of the peak remained identical to the AP-wt but

224 its MA was different. Thus, similar to the single-point mutants, wide variation in the

225 heterogeneity in catalytic activities was observed. This high heterogeneity in the

226 highly active AP mutants might be due to structural heterogeneity involving the active

227 site, such as the flipping of catalytically important residues or a large change in

228 phosphate coordination as demonstrated by previous studies ${ }^{38-41}$. In the future, 
229 further elucidation of the direct correlation between structural changes in the active

230 site and the alteration of functional substates is pivotal to our understanding of

231 enzyme mechanism and evolution.

233 Functional heterogeneity and structural position of mutations In order to investigate whether AP possesses mutational hot spots that may be responsible for enhanced activity and/or high functional heterogeneity, we plotted the $M A$ and $C V_{n}$ of each single mutation against the distance of $C \alpha$ of mutation sites

237 from phosphate in active site (Fig. $\mathbf{2} \mathbf{b}$ and $\mathbf{c}$ ). We also presented a heat map of the crystal structure of AP-wt in order to map mutation sites with higher $M A$ and $C V_{n}$ on

239 the 3D structure (Supplementary Fig. 8). Interestingly, the mutations giving rise to

240 highly active mutants were located in the vicinity of the active site; however, there

241 was no significant correlation between the $C V_{n}$ and the distance from the active site.

242 We also analyzed mutations based on their proximity to the two disulfide bonds

243 (C168-C178 and C286-C336) or the interface between the monomers

244 (Supplementary Fig. 9) ${ }^{42,43}$, but there were no clear correlations. Thus, these

245 findings suggest that functional heterogeneity can potentially be modulated globally

246 across the whole enzyme structure. 


\section{Functional heterogeneity and promiscuity}

It has been suggested that the expansion of functional substates may be

250 related to the enhancement of reactivity to promiscuous substrates ${ }^{3,17-20,22,24,34}$. To

251 test this, we measured the activity of AP-wt and four highly active mutants (D101S,

252 G118D, D153S, and K328R) against promiscuous substrates: phosphate diesters

253 (bis-p-nitrophenyl phosphate, bpNPP) and phosphonate monoesters ( $p$-nitrophenyl

254 phenyl phosphonate, pNPPP), in addition to another phosphate monoesters

255 substrate, $p$-nitrophenyl phosphate ( $p$ NPP) (Fig. 4a). Then, we analyzed the

256 correlation between $C V_{n}$ measured using FDP and the promiscuous activities.

257 bpNPP and pNPPP can be employed to evaluate the promiscuous activity of AP-

$258 \mathrm{wt}^{44,45}$. Since single-point mutations involving the active site can significantly alter

259 promiscuous activity, including $E$. coli $\mathrm{AP}^{46-48}$, those highly active mutants could be

260 used to assess the effect of $C V_{n}$ levels (9-35\%) on promiscuity. The activities against

$261 b p N P P$ and $p$ NPPP were too low to be measured at the single-molecule level; hence,

262 we measured their promiscuous activities in a bulk solution with purified enzymes

263 (Supplementary Fig. 3c). Although the four AP mutants exhibited higher activity

264 against pNPP than the AP-wt (Fig. 4b), no correlation was observed between

265 catalytic activity and $C V_{\mathrm{n}}$ (Fig. 4c, $R^{2}=0.28$ ), as previously observed with FDP.

266 Interestingly, however, D153S and K328R, which have higher $C V_{\mathrm{n}}$, exhibited greater 
267 activity than the AP-wt against promiscuous substrates (Fig. 4b). In particular,

268 D153S, which displayed the highest $C V_{\mathrm{n}}$ among the mutants, showed 4- and 6-fold

269 higher activity against bpNPP and pNPPP than the AP-wt. On the other hand,

270 D101S and G118D, whose $C V_{n}$ s were close to AP-wt, showed only modest activity

271 enhancement. To investigate a possible correlation between functional heterogeneity

272 and promiscuity, the activities of the mutants toward promiscuous substrates were

273 plotted against the $C V_{n}$ of the mutants (Fig. 4c). Clear correlations were found for

274 activity against bpNPP $\left(R^{2}=0.91\right)$ and pNPPP $\left(R^{2}=0.82\right)$ (Fig. 4c). These

275 observations suggested that functional heterogeneity is associated with AP enzyme

276 promiscuity.

277

278 Discussion

279 In the present study, we assessed the functional heterogeneity of a model

280 enzyme using AP-wt and 69 mutants by performing single-molecule assays with the

281 FRAD. AP-wt showed the lowest level of functional heterogeneity, and most of the

282 mutants showed more significant heterogeneity than the AP-wt. These observations

283 showed that even a single-point mutation in AP-wt is prone to enhancing functional

284 heterogeneity, suggesting that AP-wt is perhaps optimized to reduce functional

285 heterogeneity. A possible scenario is that AP has evolved as a specialist enzyme, 
286 minimizing the functional substate toward its native function of phosphate

287 monoesterase. Native enzymes have undergone a long evolutionary process under

288 various selection pressures from diverse environmental conditions; thus, these

289 natural enzymes may have undergone optimization in terms of their conformational

290 and functional substates ${ }^{21-24}$. If the functional substates of AP-wt for its native

291 function have been evolutionary optimized over billions of years, mutations may

292 readily disrupt the fine-tune balance of the substates. As a results, the expansion of

293 the functional substates may lead to high functional heterogeneity in most mutants

294 independent of catalytic activity toward FDP or pNPP (Fig. 3 and 4c).

296 functional heterogeneity and promiscuity (Fig. 4b and C). AP-wt is highly optimized

297 for the hydrolysis of phosphate monoesters so that AP-wt has low promiscuous

298 activity, $>10^{9}$ lower than the native AP activity ${ }^{44,45}$, against non-native substrates with

299 phosphate diesters or phosphonate monoesters. Interestingly, an active mutant,

300 D153S, exhibited higher functional heterogeneity and remarkable enhancements of

301 catalytic activity against non-native substrates with bpNPP and $p$ NPPP than the AP-

302 wt. Similarly, evolved GUS with high promiscuous activity against several glycosidic

303 substrates exhibited more extensive heterogeneity in catalytic activity than the wild-

304 type enzyme ${ }^{34}$. Moreover, wild-type GAL showed higher heterogeneity in catalytic 
305 activity than wild-type GUS because it retains three catalytic functions ${ }^{34,49}$. These

306 findings and our results reinforce the long-discussed concept of enzyme evolution;

307 enzymes can enhance their promiscuity or multi-functionality by gaining functional

308 heterogeneity with multiple functional substates. However, only a handful of

309 enzymes and their variants have been measured at the level of single enzyme

310 kinetics to date, and it is difficult to generalize these observations to other systems.

311 For further generalization of this scenario, more comprehensive studies involving the

312 various types of enzymes are required. 
315 Materials and Methods

316 Creation and cloning of AP for single-molecule assay study $^{36}$. Each site was mutagenized using NNK degenerate codons giving rise to 450 individual libraries (one for each position), then subcloned into a T7 expression

321 vector with an ampicillin selective marker (pRSET-B) (Invitrogen ${ }^{\mathrm{TM}}$, Thermo Fisher

322 Scientific, Waltham, MA, USA) by Gibson assembly. The mutants used in the single-

323 molecule assays were randomly selected from the library. The plasmid library for

324 each selected position was transformed into HIT-JM109 competent cells (RBC

325 Bioscience, Birmingham, U.K.), and the cells were plated on LB agar plates

326 containing $100 \mu \mathrm{g} / \mathrm{mL}$ ampicillin. After culturing the cells overnight, three to ten

327 colonies were picked for each selected position and cultured separately in the LB

328 media overnight. The plasmids were then purified from the cultured cells and

329 mutation sites were confirmed by DNA sequencing. For each mutation position, one

330 to eight plasmids were obtained using this method, and one plasmid was randomly

331 selected from those plasmids and used for expression with a PURE system 
332 (PURExpress In Vitro Protein Synthesis Kit, New England BioLabs, Ipswich, MA, 333 USA). Of the highly active mutant APs, D101S was kindly gifted by Abbott

335 Laboratories $^{38}$. The three remaining highly active mutants (G118D, D153S, and

$336 \mathrm{~K} 328 \mathrm{R})$ were obtained by performing a directed evolution starting from the library

337 described above and a random mutagenesis library constructed by error-prone PCR

338 (Agilent, Santa Clara, CA, USA). We used a FRAD to perform directed evolution

339 under identical conditions to our previous study ${ }^{36}$. As the previous 65 variants, these

340 four mutants were also subcloned into the pRSET-B vector for expression.

342 Expression of AP for the single-molecule assay

344 PURE system. The PURE system includes all proteins required for transcription and

345 translation, and proteins can be expressed by mixing the PURE system and DNA

346 that encodes the sequence of proteins downstream of a T7 promoter. To express the

347 APs, PURE components $A$ and $B$, disulfide bonding enhancer 1 and 2 (New England

348 BioLabs), RNase inhibitor (Takara Bio Inc., Shiga, Japan), $100 \mu \mathrm{M} \mathrm{ZnCl}$, and 50

$349 \mathrm{ng}$ of the pRSET-B expression vector were mixed by vortexing and incubated at 
$351 \mathrm{ZnCl}_{2}$ promotes dimer formation of fully activated APs without loss of their bonds and

352 zinc ions ${ }^{16}$. Note that the levels of endogenous AP in the PURE system was

353 negligible, and thus, the expressed AP could be directly used for measurements

354 without purification after dilution to an optimum concentration ${ }^{16}$.

Expression and purification of AP for bulk reaction

For bulk reactions, a vector $(\mathrm{pET}-21(+))$ for large-scale expression and

purification in E. coli was custom-synthesized (Twist Bioscience, South San

359 Francisco, CA, USA). The pET-21(+) vector was designed to have a signal peptide ${ }^{50}$

360 at the N-terminus and a Strepll-tag on the C-terminus. The sequences of AP-wt,

361 D101S, G118D, D153S, and K328R were inserted between the signal peptide and

362 the Strepll-tag.

AP-wt and highly active mutants were expressed using OverExpress

364 C43(DE3) cells. The pET-21(+) expression vector bearing the AP sequence was

365 transformed into cells by electroporation. The transformed cells were pre-cultured

366 overnight at $37^{\circ} \mathrm{C}$ in $8 \mathrm{~mL}$ of the LB media containing $100 \mu \mathrm{g} / \mathrm{mL}$ ampicillin. Then, 2

$367 \mathrm{~mL}$ of the culture solution was transferred to $200 \mathrm{~mL}$ of Overnight Express Instant TB

368 medium (MilliporeSigma, Burlington, MA, USA) in a $2 \mathrm{~L}$ flask and cultured at $37^{\circ} \mathrm{C}$ for

$3698 \mathrm{~h}$, and then the AP protein was expressed at $30^{\circ} \mathrm{C}$ for $16 \mathrm{~h}$. The cell lysis steps 
370 followed a previously established osmotic shock method ${ }^{41}$. The cell lysate passed

371 over a Strep-Tactin column (IBA Lifesciences, Goettingen, Germany) pre-

372 equilibrated with a binding buffer (10 mM Tris- $\mathrm{HCl}(\mathrm{pH}$ 7.4), $200 \mathrm{mM} \mathrm{NaCl}$, and 10

$373 \mu \mathrm{M} \mathrm{ZnCl}$ ) and washed with ten column volumes of the clean binding buffer. Then,

374 proteins were eluted using the elution buffer $(20 \mathrm{mM}$ biotin, $10 \mathrm{mM}$ Tris- $\mathrm{HCl}(\mathrm{pH} 7.4)$,

$375200 \mathrm{mM} \mathrm{NaCl}$, and $10 \mu \mathrm{M} \mathrm{ZnCl}_{2}$ ), which was subsequently exchanged to a storage

376 buffer (10 mM sodium MOPS, pH 7.0, $50 \mathrm{mM} \mathrm{NaCl}, 200 \mu \mathrm{M} \mathrm{ZnCl}$, and $1 \mathrm{mM} \mathrm{MgCl}$ )

377 using a desalting column (Econo-Pac 10DG, BioRad, Hercules, CA, USA). Finally,

378 the protein solutions were concentrated by centrifugation (Spin filters 10k, Pall

379 Laboratory, Port Washington, NY, USA), and their purities were analyzed by SDS-

380 PAGE (MilliporeSigma).

381

382 Fabrication of FRAD

The FRAD was fabricated on a cover glass via photolithography and

384 etching $^{16,35-37}$. The cover glass $(24 \times 32 \mathrm{~mm}$, thickness $0.13-0.17 \mathrm{~mm}$, Matsunami

385 Glass Ind., Osaka, Japan) was sonicated in $10 \mathrm{M} \mathrm{KOH}$ solution (FUJIFILM Wako

386 Pure Chemical Industries, Osaka, Japan) for $10 \mathrm{~min}$. After washing with deionized

387 water and drying under a nitrogen stream, an amorphous fluorocarbon polymer

388 (CYTOP, Asahi Glass, Tokyo, Japan) was coated onto the glass at 4,000 rpm for 30 
389 s using a spin coater. The glass was then baked at $180^{\circ} \mathrm{C}$ for $30 \mathrm{~min}$. A positive

390 photoresist (AZ-4903, Merck KGaA, Darmstadt, Germany) was coated onto the

391 CYTOP-coated glass at $7,500 \mathrm{rpm}$ for $60 \mathrm{~s}$. The glass was baked at $55^{\circ} \mathrm{C}$ for $3 \mathrm{~min}$ and at $110^{\circ} \mathrm{C}$ for $10 \mathrm{~min}$. Photolithography was then performed using a mask aligner

393 (BA100it, Nanometric Technology, Tokyo, Japan) with a chrome photomask with 3

$394 \mu \mathrm{m}$ holes. The photoresist on the glass was then developed using a photoresist

395 developer (AZ300 MIF, Merck KGaA) for 5 min. The patterned glass coverslip was

396 washed with deionized water. After drying on a hot plate, the glass was etched by $\mathrm{O}_{2}$

397 plasma using a reactive ion etching machine (RIE-10NR, Samco, Santa Clara, CA,

398 USA). Then, the remaining photoresist on the glass was entirely removed using

399 acetone, and the glass was rinsed with 2-propanol and deionized water. The

400 diameter and thickness of the reactor were measured using a 3D laser scanning

401 microscope (VK-X200, KEYENCE, Osaka, Japan). The diameter and thickness of

402 the reactor array used in these experiments were $2.5-3.0 \mu \mathrm{m}$ and $0.45-0.50 \mu \mathrm{m}$, 403 respectively.

405 Reagents and solutions for the measurement of catalytic activity

406 To measure the activity of $A P$ in the bulk solution or FRAD, $1 \mathrm{M}$

407 diethanolamine (DEA) buffer (pH 9.2) (FUJIFILM Wako Pure Chemical Industry) was 
408 used as a reaction buffer ${ }^{16,35-37}$. To maximize enzyme activity, $\mathrm{ZnCl}_{2}$ and $\mathrm{MgCl}_{2}$

409 (FUJIFILM Wako Pure Chemical Industry) were added to the reaction buffer (final

410 concentrations of $\mathrm{ZnCl}_{2}$ and $\mathrm{MgCl}_{2}$ were $100 \mu \mathrm{M}$ ) ${ }^{35}$. A stock solution of FDP (AAT

411 Bioquest, Sunnyvale, CA, USA) in double-distilled water $\left(\mathrm{ddH}_{2} \mathrm{O}\right), p N P P$

412 (MilliporeSigma) in $d_{d d} \mathrm{H}_{2} \mathrm{O}, \quad b p N P P$ (MilliporeSigma) in $d_{d d} \mathrm{O}, \quad p N P P P$

413 (MilliporeSigma) in dimethyl sulfoxide (DMSO), and fluorescein dye (FUJIFILM Wako

414 Pure Chemical Industry) in DMSO were stocked at $-20^{\circ} \mathrm{C}$ and only diluted to $1 \mathrm{mM}$

415 prior to the measurement.

416

417 Single-molecule assays with FRAD

419 min. After sonication, the reactor array was thoroughly washed with excess $d_{d d} \mathrm{H}_{2} \mathrm{O}$

420 and dried on a hot plate. The flow channel was assembled on the surface of the

421 FRAD with double-sided adhesive tape (TERAOKA SEISAKUSHO, Tokyo, Japan)

422 and a custom-made upper glass, which has inlet and outlet holes for solution and oil

423 flow to the FRAD (Supplementary Fig. 1a) $)^{16,35-37}$. The upper glass was coated with

424 CYTOP to reduce the affinity of the surface for the water solution. The double-sided

425 tape was cut out in the shape of the flow path using a cutting machine (STIKA,

426 Roland DG, Shizuoka, Tokyo), and the tape was applied to the surface of the FRAD. 
427 Then, the upper glass was applied to the tape, and the FRAD was degassed for tight

428 binding. The reaction solution $(10 \mu \mathrm{L})$ was prepared by mixing AP and a fluorogenic

429 substrate (FDP) in the DEA buffer on ice. Then, the reaction solution was added to

430 the flow channel by pipetting (Supplementary Fig. 1b), and the air in the reactor

431 was completely degassed by sonication for $1 \mathrm{~s}$ in a water bath. Fluorinated oil (FC-

432 40, Merck, Kenilworth, NJ, USA) was used to remove the excess amounts of the

433 reaction solution and to seal the reactor (Supplementary Fig. 1b). Measurements

434 commenced 5 min after the reactor was sealed. New flow channel of FRAD was used for each assay.

436

437 Fluorescence microscopy for single-molecule assay

All images of single-molecule assays were acquired using an inverted

439 fluorescence microscope (Eclipse Ti2, Nikon, Tokyo, Japan) equipped with a $40 \times$

440 objective lens (Nikon, Plan Apo, NA = 0.9), multi-wavelength LED illumination (X-cite

441 Turbo, Excelitas, Waltham, MA, USA), sCMOS camera (Zyla, Andor Technology,

442 Belfast, UK), an incubator for temperature control (TOKAI HIT Co., Ltd., Shizuoka,

443 Japan), and a Perfect Focus System (Nikon). The fluorescence of the fluorescein

444 dye was measured using filter sets (excitation 480/15 nm, dichroic $505 \mathrm{~nm}$, emission

$445535 / 20 \mathrm{~nm}$, Nikon). All measurements were performed using the NIS-Element 
446 software (Nikon). In end-point measurements, 90 different fields of view were taken

447 with $100 \mathrm{~ms}$ exposure time. The temperature of the incubator was set to $28^{\circ} \mathrm{C}$ for all

448 measurements.

449 The leakage of the fluorophore (i.e., the reaction product) from the reactor

450 was evaluated by the photobleaching of a single reactor (Supplementary Fig. 2).

451 Photobleaching was performed using a confocal microscope (Eclipse Ti-E

452 microscope, Nikon) equipped with a $60 \times$ objective lens (Plan Apo, NA $=1.40$, Nikon)

453 and a blue laser (488 $\mathrm{nm}$, Coherent). Photobleaching and time-course

454 measurements were performed using NIS-Element software.

455

456 Analysis of single-molecule assay data

Fluorescent images of FRAD were analyzed using a custom Python code.

458 Fluorescence intensity histograms were plotted after analyzing the fluorescence

459 intensity in the FRAD (Fig. 1c). Enzyme activity was determined as follows: the first

460 peak was fitted by a single Gaussian distribution, and the mean intensity $\left(M l_{1}\right)$ and

461 standard deviation $\left(S D_{1}\right)$ of peak 1 were analyzed. The single enzyme activity was

462 observed at a threshold where the fluorescence intensity was $>M I_{1}+5 \times S D_{1}$. Then,

463 the distribution of single enzyme activity (peak 2) was fitted by a single Gaussian

464 distribution in order to extract the mean intensity $\left(M I_{2}\right)$ and $S D\left(S D_{2}\right)$. For all mutants, 
465 distinct peak 2 separated from peak 1 was observed after more than $4 \mathrm{~h}$ of

466 measurement. The turnover number $\left(k_{\text {cat }}\left(\mathrm{s}^{-1}\right)\right)$ was calculated from $M A$ by using a

467 calibration curve providing the concentration of the fluorescein as a function of

468 fluorescence intensity in the FRAD (Supplementary Fig. 4a). $C V_{1}$ is the lower detection limit of enzyme activity heterogeneity and

470 represents systematic error of variations in the volume of each reactor and optical

471 noise created by the detection system of the microscope. In this experiment, $C V_{2}$,

472 the distribution of catalytic activities of all APs, was always $>C V_{1}$ (the mean value of

$473 C V_{1}$ in all measurements was $7 \pm 2 \%$, mean $\pm S D$ ).

In our FRAD, the number of enzyme molecules in a single reactor follows a

475 Poisson distribution ${ }^{16,35-37}$. Therefore, the probability and expected average number

476 of enzyme molecules per reactor $(\lambda)$ can be calculated using the Poisson's equation:

$$
P=\frac{e^{-\lambda} \lambda^{k}}{k !}
$$

478 where $k$ is the physical number of enzyme molecules in a reactor. $\lambda$ can be

479 calculated from the number of positive reactors, including enzyme molecules, and

480 the total number of reactors ${ }^{16,35-37}$. The mean $\lambda$ in all measurements was $0.17 \pm 0.13$

481 (mean $\pm S D$ ). With this dilution, we expected that approximately $14 \%(\sim 6,400)$ of the

482 reactors contained the desired single enzyme molecule and $\sim 2 \%(\sim 900)$ contained 
483 two enzyme molecules, and the remaining $\sim 84 \%(\sim 38,600)$ reactors were empty,

484 that is, without any enzymes.

485

486 Measurement of catalytic activity in bulk solution AP activity in the bulk solution was measured in a 384-well plate by

488 fluorometry using a plate reader (Synergy H1, BioTek, Winooski, VT, USA). For 489 measurements with FDP, the excitation and emission wavelengths were set to 488

$490 \mathrm{~nm}$ and $532 \mathrm{~nm}$, respectively. For the absorbance measurements with pNPP, bpNPP,

491 and $p$ NPPP, the wavelength was set to $405 \mathrm{~nm}$. Changes in fluorescence intensity

492 and absorbance were acquired at two-minute intervals at $28^{\circ} \mathrm{C}$. A reaction solution

$493(50 \mu \mathrm{L})$ was prepared by mixing AP and substrate in DEA buffer on ice, and then the

494 mixture was applied to the plate wells. The activity was measured after 5 min to 495 ensure temperature equilibration. Three independent measurements were taken to 496 analyze the mean and $S D$ of enzyme activity. pNPP, bpNPP, and pNPPP (1 mM)

497 were used to measure the promiscuous activity of APs. The activities of pNPP, 498 bpNPP, and pNPPP were measured for $20 \mathrm{~min}$ or $6 \mathrm{~h}$. Enzyme activity was 499 determined from a linear fit of the increase in fluorescence intensity or absorbance 500 over time. The initial rate for FDP was measured using seven different 
501 concentrations and fitted by the Michaelis-Menten equation using KaleidaGraph

502 (Synergy Software, Reading, PA, USA).

503 
504 Acknowledgements:

505 The authors are grateful to Kenji Akama and Naoki Soga for their technical

506 assistance and to Toshiharu Suzuki and members of the Tokuriki lab for helpful

507 discussions and revision of the manuscript. This work was supported by the ImPACT

508 Program of the Council for Science, Technology, and Innovation, Japan Science and

509 Technology Agency (JST) (to H.N.), Grant-in-Aid for Scientific Research on

510 Innovation Areas from the Japan Society for the Promotion of Science (JSPS)

511 (JP19H05380 to H.U.), Grant-in-Aid for Scientific Research (S) from JSPS

512 (JP19H05624 to H.N.), CREST program from JST, (JPMJCR19S4 to H.N.), and

513 Human Frontier Science Program (HFSP) Program Grant (RGP0054/2020) (to H.N

514 and N.T.).

515

516 Author Contributions

517 M.S., H.U., N.T., and H.N. designed the experiments; M.S., S.H., and K.M.

518 performed experiments and analyzed the data; M.S., N.T., and H.N. wrote the paper.

519

520 Competing interests

521 The authors declare no competing interests.

\section{References}


1. Henzler-Wildman, K. \& Kern, D. Dynamic Personalities of Proteins. Nature 450, 964-972 (2007). 789-796 (2009)

3. Khersonsky, O. \& Tawfik, D. S. Enzyme Promiscuity: A Mechanistic and Evolutionary Perspective. Annu. Rev. Biochem. 79, 471-505 (2010). Folded States. Nat. Struct. Mol. Biol. 11, 338-345 (2004).

5. Tuinstra, R. L. et al. Interconversion Between Two Unrelated Protein Folds in (2008). 
7. Alexander, P. A., He, Y., Chen, Y., Orban, J. \& Bryan, P. N. A Minimal Sequence Code for Switching Protein Structure and Function. Proc. Natl Acad. Sci. U. S. A. 106, 21149-21154 (2009). 20, 482-488 (2010).

9. Si, Z. et al. Different Functional States of Fusion Protein gB Revealed on Human Cytomegalovirus by Cryo Electron Tomography with Volta Phase Plate. PLOS Pathog. 14, e1007452 (2018).

10. Xue, Q. \& Yeung, E. S. Differences in the Chemical Reactivity of Individual Molecules of an Enzyme. Nature 373, 681-683 (1995).

12. Hinczewski, M., Hyeon, C. \& Thirumalai, D. Directly Measuring Single-

11. English, B. P. et al. Ever-fluctuating Single Enzyme Molecules: MichaelisMenten Equation Revisited. Nat. Chem. Biol. 2, 87-94 (2006). Molecule Heterogeneity Using Force Spectroscopy. Proc. Natl Acad. Sci. U. S. A. 113, E3852-E3861 (2016). 
13. Galenkamp, N. S., Biesemans, A. \& Maglia, G. Directional Conformer Exchange in Dihydrofolate Reductase Revealed by Single-Molecule Nanopore Recordings. Nat. Chem. 12, 481-488 (2020). States of Single Enzyme Molecules. J. Am. Chem. Soc. 130, 5349-5353 (2008).

15. Jiang, Y. et al. Single-molecule Mechanistic Study of Enzyme Hysteresis. ACS Cent. Sci. 5, 1691-1698 (2019). Molecules for Quantitative Digital Bioassay. Protein Sci. 30, 1628-1639 (2021).

17. Dishman, A. F. et al. Evolution of Fold Switching in a Metamorphic Protein. Science 371, 86-90 (2021). Drives Evolutionary Innovation. Science 359, 1542-1545 (2018). 
19. Sikosek, T., Krobath, H. \& Chan, H. S. Theoretical Insights into the Biophysics e1004960 (2016) Protein Bi-stability Landscapes can Potentially Resolve Adaptive Conflicts. PLOS Comput. Biol. 8, e1002659 (2012). Phenotype. PLOS Comput. Biol. 15, e1006648 (2019). 203-207 (2009). Chem. Biol. 12, 890-891 (2016). 20180330 (2018). 
25. Agarwal, P. K., Doucet, N., Chennubhotla, C., Ramanathan, A. \& Narayanan, Catalysis. Methods Enzymol. 578, 273-297 (2016). Protein Conformational Populations and Functionally Relevant Substates. Acc. Chem. Res. 47, 149-156 (2014).

27. Kaltenbach, M., Jackson, C. J., Campbell, E. C., Hollfelder, F. \& Tokuriki, N. Reverse Evolution Leads to Genotypic Incompatibility Despite Functional and Active Site Convergence. eLife 4, 1-20 (2015). 28. Baier, F. et al. Cryptic Genetic Variation Shapes the Adaptive Evolutionary Potential of Enzymes. eLife 8, (2019). (2008). 

Heterogeneous with Respect to the Activity of Individual Molecules. Can. J. Chem. 76, 623-626 (1998). Phosphatase Support Different Catalytic Rates at Room Temperature. Luminescence 17, 15-18 (2002). 282, 1877-1882 (1998). the Functional Diversity of In Vitro Evolved $\beta$-glucuronidase. J. Am. Chem. Soc. 136, 5949-5955 (2014). Using Alkaline Phosphatase with a Cumarin-based Fluorogenic Substrate. Analyst 140, 5065-5073 (2015). 
37. Honda, S., Minagawa, Y., Noji, H. \& Tabata, K. V. Multidimensional Digital Bioassay Platform Based on an Air-Sealed Femtoliter Reactor Array Device. Anal. Chem. 93, 5494-5502 (2021) Phosphatase with Higher Catalytic Activity. Protein Eng. 5, 605-610 (1992). Biochemistry 34, 13967-13973 (1995). and 328 in Escherichia coli Alkaline Phosphatase Provide Insight Towards the Structure and Function of Mammalian and Yeast Alkaline Phosphatases. J. Mol. Biol. 253, 604-617 (1995). 
634

635

636

638

639

640

641

642

643

644

645

646

42. Sone, M., Kishigami, S., Yoshihisa, T. \& Ito, K. Roles of Disulfide Bonds in Bacterial Alkaline Phosphatase. J. Biol. Chem. 272, 6174-6178 (1997).

43. Boulanger, R. R. \& Kantrowitz, E. R. Characterization of a Monomeric Escherichia coli Alkaline Phosphatase Formed Upon a Single Amino Acid Substitution. J. Biol. Chem. 278, 23497-23501 (2003).

44. O’Brien, P. J. \& Herschlag, D. Functional Interrelationships in the Alkaline Phosphatase Superfamily: Phosphodiesterase Activity of Escherichia coli Alkaline Phosphatase. Biochemistry 40, 5691-5699 (2001).

45. Pabis, A. \& Kamerlin, S. C. L. Promiscuity and Electrostatic Flexibility in the Alkaline Phosphatase Superfamily. Curr. Opin. Struct. Biol. 37, 14-21 (2016).

46. Schmidt, D. M. Z. et al. Evolutionary Potential of (beta/alpha)8-barrels: Functional Promiscuity Produced by Single Substitutions in the Enolase Superfamily. Biochemistry 42, 8387-8393 (2003).

47. Gould, S. M. Q. \& Tawfik, D. S. Directed Evolution of the Promiscuous Esterase Activity of Carbonic Anhydrase II. Biochemistry 44, 5444-5452 (2005). 
650 48. López-Canut, V., Roca, M., Bertrán, J., Moliner, V. \& Tuñón, I. Promiscuity in Alkaline Phosphatase Superfamily. Unraveling Evolution Through Molecular Simulations. J. Am. Chem. Soc. 133, 12050-12062 (2011).

653 49. Juers, D. H., Matthews, B. W. \& Huber, R. E. LacZ $\beta$-galactosidase: Structure and Function of an Enzyme of Historical and Molecular Biological Importance. Protein Sci. 21, 1792-1807 (2012). 
a

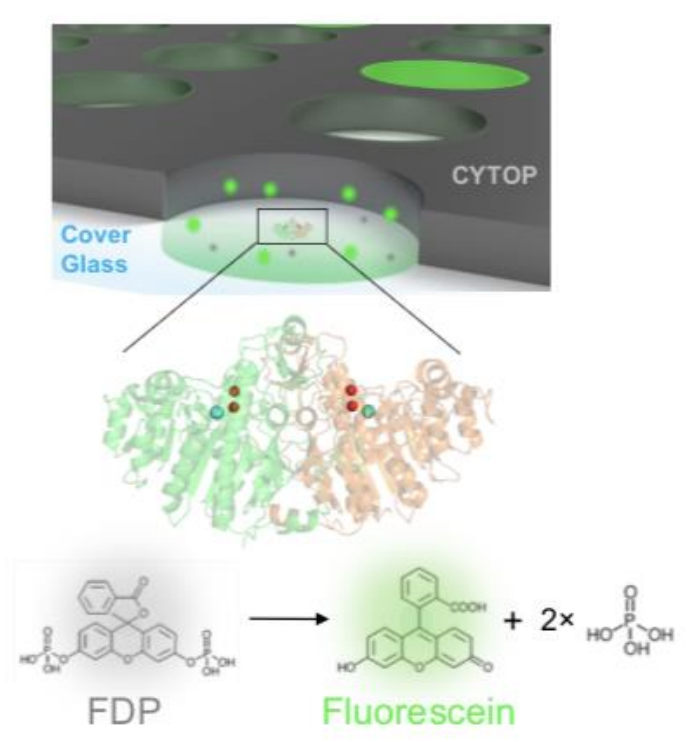

b
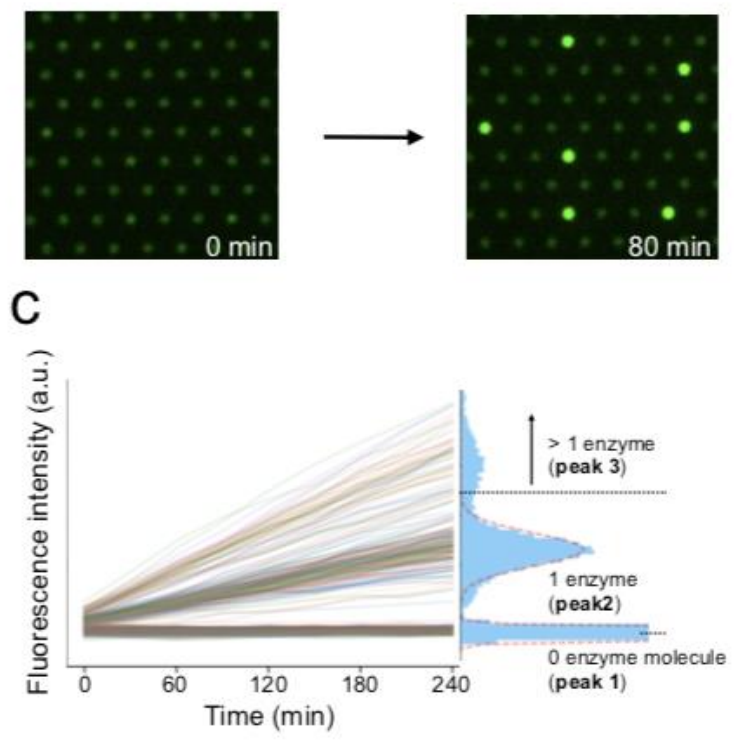

660 Figure 1. FRAD for measuring single enzyme activity. (a) Schematic image of 661 single-molecule assay in the FRAD. In each well or "reactor" patterned on 662 amorphous perfluoro polymer (CYTOP), a single AP molecule hydrolyzes non663 fluorescent FDP substrate molecules to release fluorescent product molecules 664 (fluorescein), which accumulates in the reactor. Red and magenta spheres in the 665 crystal structure of dimeric AP-wt (PDB ID: 1ED8) represent $\mathrm{Zn}^{2+}$ and $\mathrm{Mg}^{2+}$ ions in 666 the active site. (b) Snapshots of time-course measurements of catalytic activities 667 observed by microscopy. Reactors containing AP-wt molecules exhibit increase in 668 fluorescence intensity. (c) Typical time-course measurement of fluorescence 669 intensity in reactors. The histogram shows the distribution of fluorescence intensity at $670240 \mathrm{~min}$. The histogram typically presents three peaks: peak 1 (enzyme-free 671 reactors), peak 2 (single enzyme activity), and peak 3 (containing two or more 672 enzyme molecules in a single reactor). The dotted red lines indicate fitting with 673 Gaussian functions.

674 
a
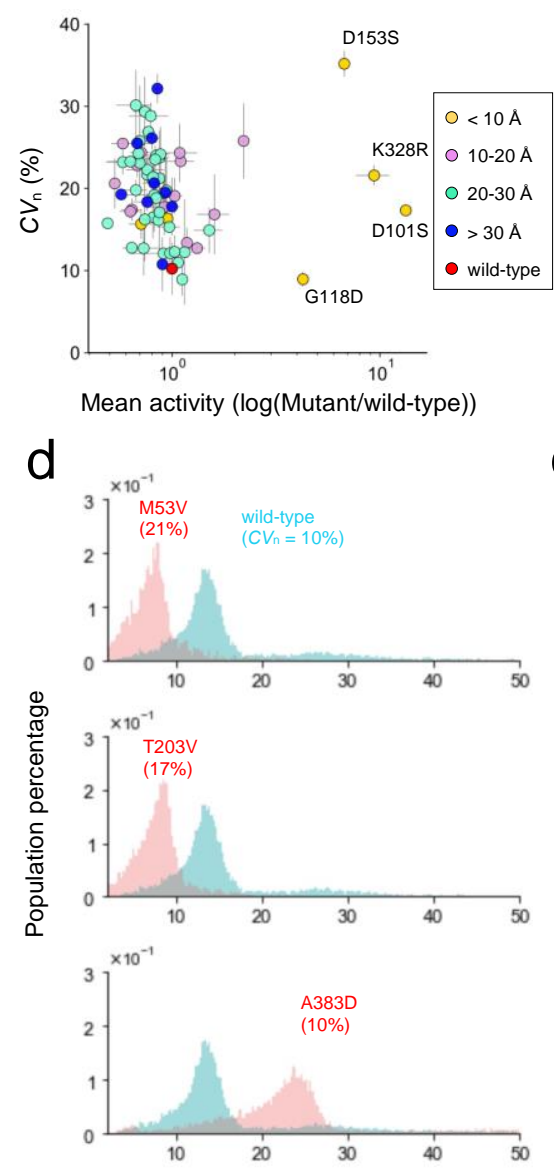

$\mathrm{b}$

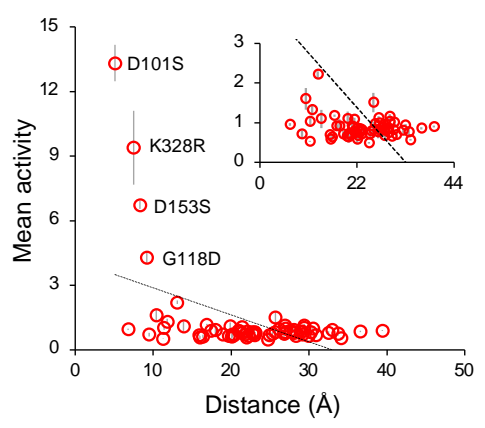

e
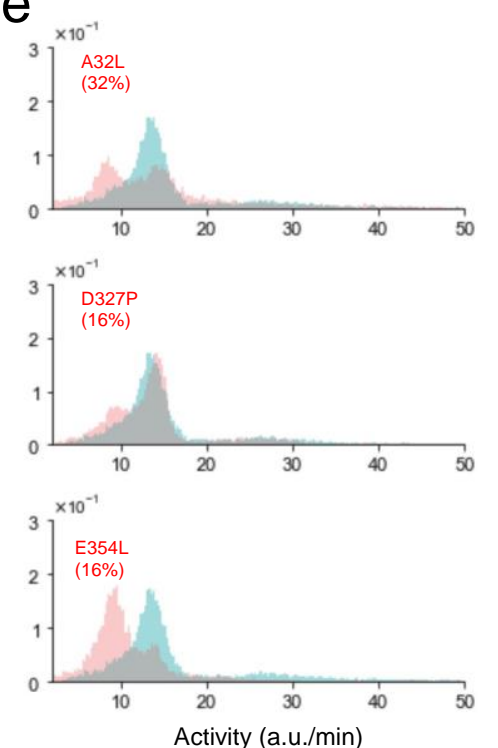

C
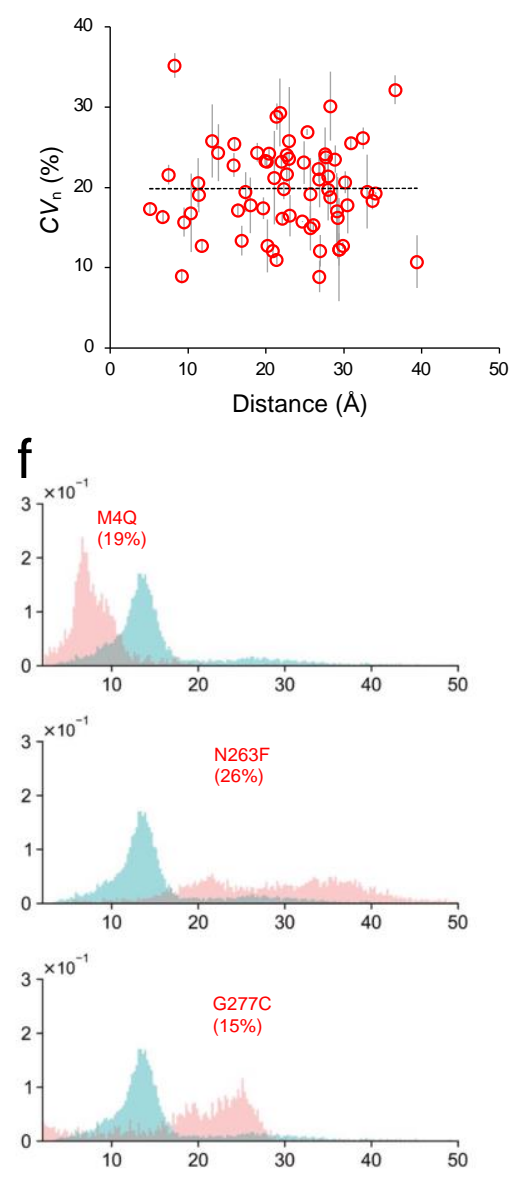

676 Figure 2. Alteration in catalytic activity and functional heterogeneity by single-
677 point mutations. (a) Mean catalytic activity $(M A)$ (a.u./min) and functional

678 heterogeneity $\left(C V_{n}\right)(\%)$ of AP-wt and mutant APs were plotted (means $\left.\pm S D\right)$. The

679 activity of the mutants was normalized to that of the AP-wt, and the x-axis was

680 converted to a logarithmic scale. The data points are colored according to the

681 distance $(\AA)$ between a given mutation and the ligand (phosphate) present in the

682 active site to the crystal structure of AP. The red-filled circle represents the AP-wt.

683 (b) Dependence of activity on distance from the ligand to mutation sites. The dotted

684 line shows a linear fitting of the plot (slope $=-0.13, R^{2}=0.24$ ). (c) Dependence of

$685 C V_{n}$ on the distance from ligand to mutation sites. The dotted line shows a linear

686 fitting of the plot (slope $\left.=-0.01 \times 10^{-1}, R^{2}=0.05 \times 10^{-4}\right)$. (d) -(f) Representative

687 distributions of mutants that exhibited single peaks shifted from the AP-wt (d),

688 additional peaks partially overlapping with the AP-wt (e), and shifted additional peaks

689 (f).

690 


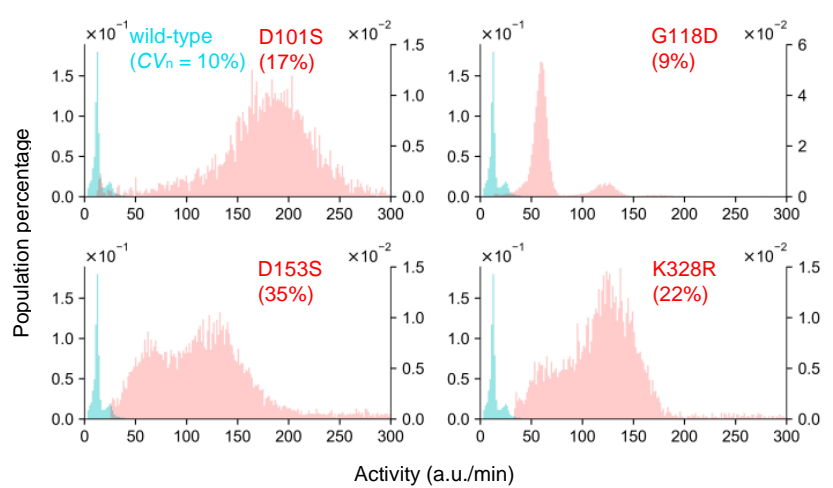

692 Figure 3. Alteration of catalytic activity and functional heterogeneity in highly

693 active mutants. The distribution of catalytic activity of AP-wt and highly active AP

694 mutants (D101S, G118D, D153S, and K328R). Blue and red bins show the

695 distribution of the AP-wt (first y-axis) and mutants (second y-axis), respectively.

696 

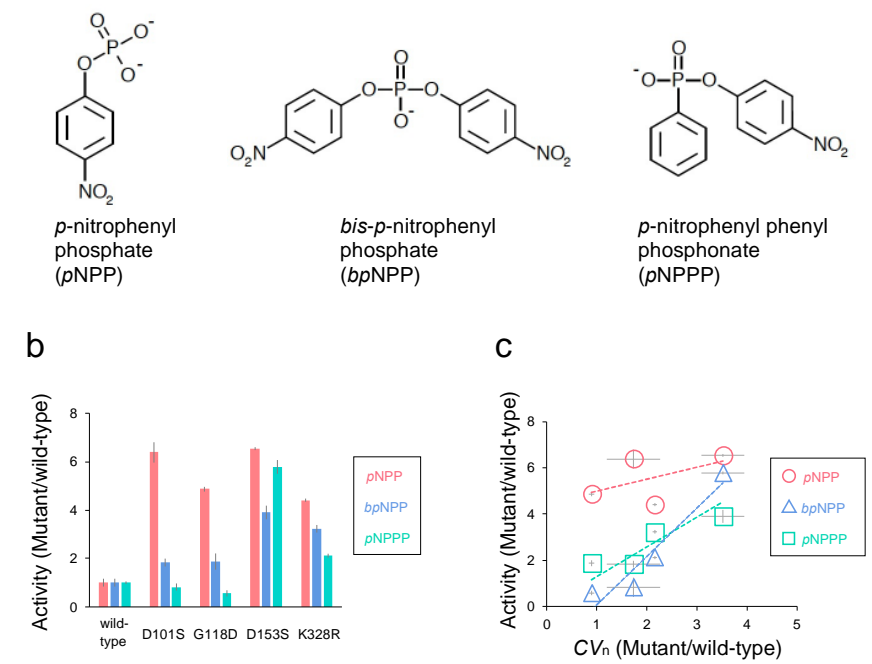

698 Figure 4. Correlation between functional heterogeneity and catalytic activity

699 against promiscuous substrates. (a) Chemical structures of phosphate

700 monoesters ( $p$-nitrophenyl phosphate, $p N P P$ ), phosphate diesters (bis- $p$-nitrophenyl

701 phosphate, bpNPP), and phosphonate monoesters ( $p$-nitrophenyl phenyl

702 phosphonate, pNPPP) substrates. (b) Comparative activity of the AP-wt and highly

703 active mutant APs (D101S, G118D, D153S, and K328R) against the substrates in

704 three independent measurements (means $\pm S D$ ) in the bulk solution. The activity of

705 mutants was normalized to that of the AP-wt. (c) Correlation between activities

706 against those substrates and $C V_{\mathrm{n}}$ of the highly active mutants. Activity and $C V_{\mathrm{n}}$ of

707 mutant AP were comparable to those of AP-wt. The $C V_{\mathrm{n}}$ of the mutants was

708 measured using the FDP shown in Fig. 3. The slopes of $p N P P, b p N P P$, and pNPPP were $0.51\left(R^{2}=0.28\right), 2.10\left(R^{2}=0.91\right)$, and $0.85\left(R^{2}=0.82\right)$, respectively. 
a

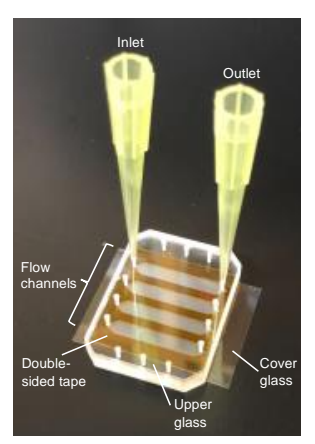

b

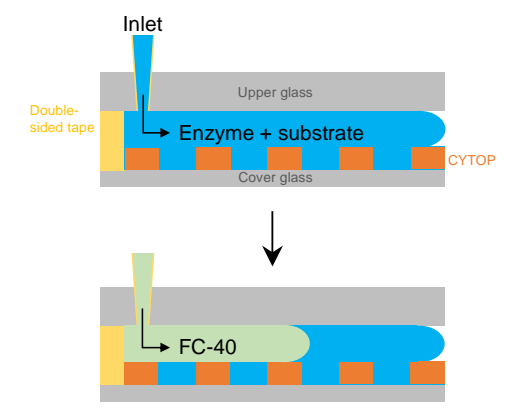

712 Supplementary Figure 1. Set up of FRAD for single-molecule assay. (a) The

713 flow channel was assembled on the FRAD. (b) Enzyme assay mixture was

714 injected into the flow channel, and then, FC-40 was applied to displace the assay

715 mixture and seal the reactors.

716 
a

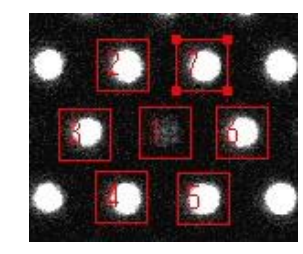

1 photobleached reactor

2-7 surrounding reactors

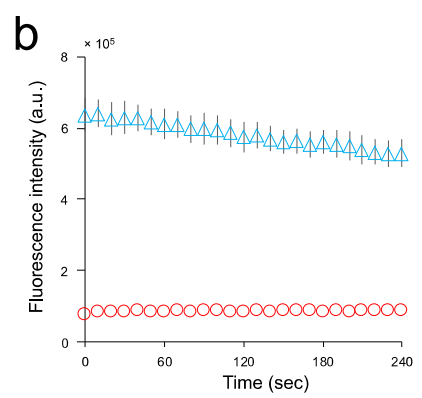

718 Supplementary Figure 2. Measuring leakage of fluorescein dye from reactors.

719 (a) One reactor (rectangle 1) was photobleached using a confocal microscope.

720 Changes in fluorescence intensity in photobleached and surrounding reactors

721 (rectangle 2-7) were simultaneously measured. (b) Time-course measurement of

722 fluorescence intensity in photobleached and surrounding reactors for $240 \mathrm{~min}$. Red

723 circles indicate fluorescence intensity change of the photobleached reactor; blue

724 triangles represent mean fluorescence intensity and $S D$ of the surrounding reactors.

725 The reactors did not show significant fluorescence changes, indicating almost no

726 solution leakage or interchange among the reactors.

727 
a

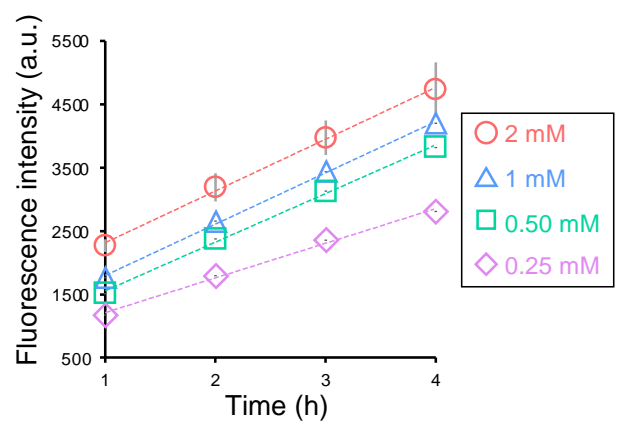

C

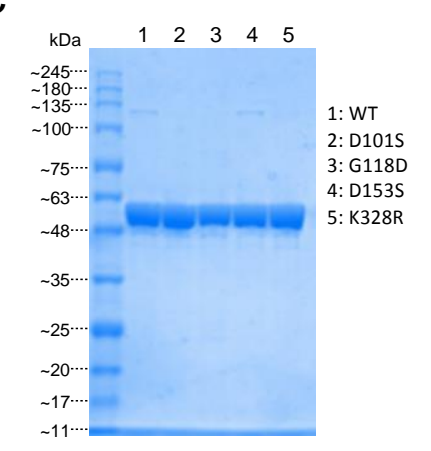

b

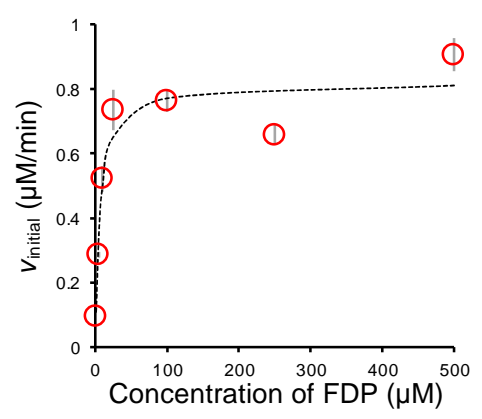

729 Supplementary Figure 3. Characterization of catalytic activity of AP-wt in FRAD

730 and bulk solution (a) Time-course measurements of AP-wt in FRAD. The

731 concentrations of FDP were $2 \mathrm{mM}$ (red circles), $1 \mathrm{mM}$ (blue triangles), $0.5 \mathrm{mM}$

732 (green rectangles), and $0.25 \mathrm{mM}$ (purple diamonds). The plot illustrates changes in

733 mean intensity $\left(M I_{2}\right)$ across three independent measurements (mean $\pm S D$ ). The

734 dotted lines in each plot show a linear fitting of the curves (slopes were 817, 810,

735768 , and 547 (a.u./h) in 2.0, 1.0, 0.5, and 0.25 mM FDP, respectively, with

$736 R^{2} \geqq 0.99$ ). (b) Catalytic activity of purified AP-wt was measured in bulk solution with

737 FDP. Red circles show mean initial velocity $\left(V_{\text {init, }} \mu \mathrm{M} / \mathrm{min}\right)$ and $S D$ in three wells. The

738 dotted line shows a fitting with the Michaelis-Menten equation. Analyzed kinetic

739 parameters, $k_{\text {cat }}$ and $K_{\mathrm{M}}$, were $27\left(\mathrm{~s}^{-1}\right)$ and $8(\mu \mathrm{M})$, respectively. (c) SDS-PAGE

740 analysis of purified AP-wt and highly active AP mutants. $20 \mathrm{ng}$ of the enzyme was

741 loaded onto the gel. Intense bands were located at the molecular weight of

742 monomeric AP between 48 and $63 \mathrm{kDa}$ corresponding to the monomeric form of AP. 

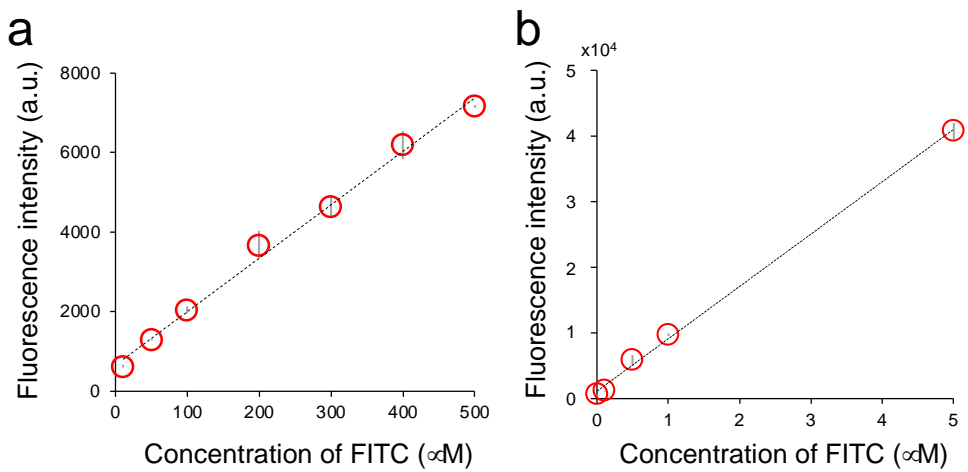

745 Supplementary Figure 4. Linear correlation between fluorescein dye

746 concentration and fluorescence intensity in FRAD (a) and 384-well plates (b).

747 (a) Red circles represent mean intensity and SD measured in two different FRADs.

748 Dotted line represents linear fitting of the curves (slope $=13$ (a.u./ $\mu \mathrm{M}), \mathrm{R}^{2}=0.99$ ).

749 (b) Red circles indicate mean intensity and SD measured in three different wells. The 750 dotted line represents a linear fitting of the curves (slope $=8,990$ (a.u. $/ \mu \mathrm{M}), \mathrm{R}^{2}=$ 751 0.99). 

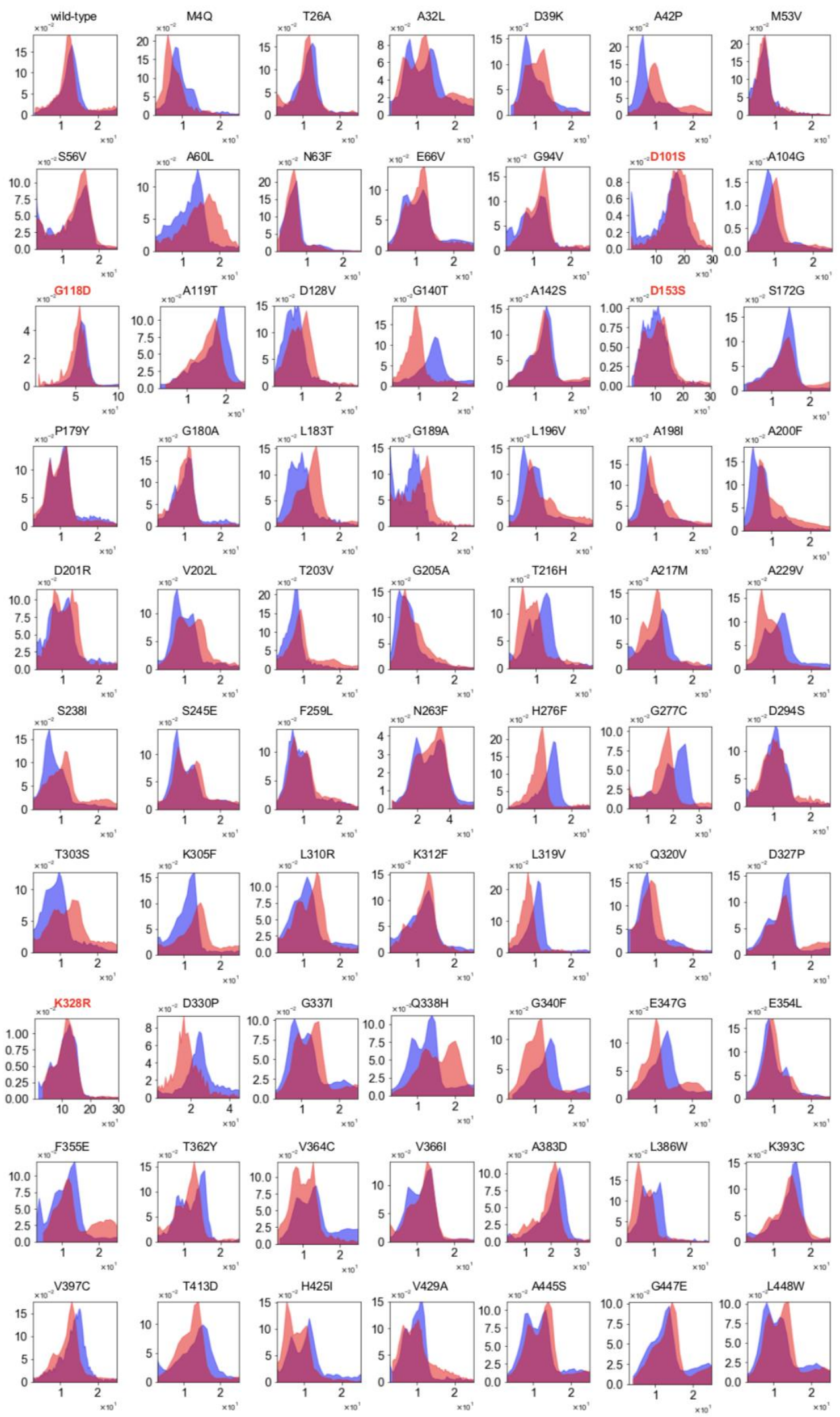

FRAD. X- and y-axes represent catalytic activities (a.u./min) and population 
756 percentage of enzyme molecules (peaks 2 and 3). Red and blue bins show the 757 distribution of two independent measurements. The identifiers of highly active 758 mutants are shown in red.

759 
a

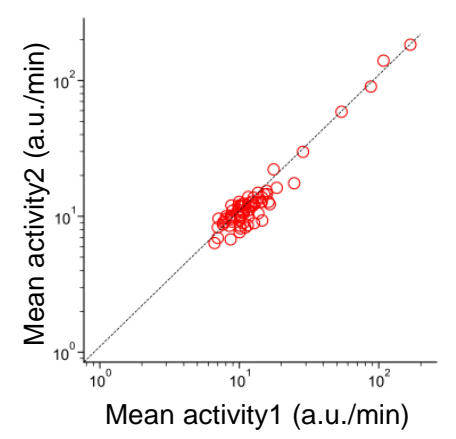

b

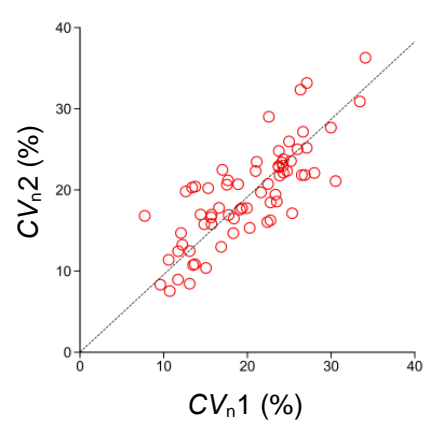

(b) represent the correlation between two independent measurements of mean

763 catalytic activity $(M A)$ and functional heterogeneity $\left(C V_{n}\right)$ of each of the mutants. The

764 dotted lines show linear fittings of the plots. The slopes of $(a)$ and $(\mathbf{b})$ were $1.1\left(R^{2}=\right.$ $7650.99)$ and $1.0\left(R^{2}=0.97\right)$, respectively. 

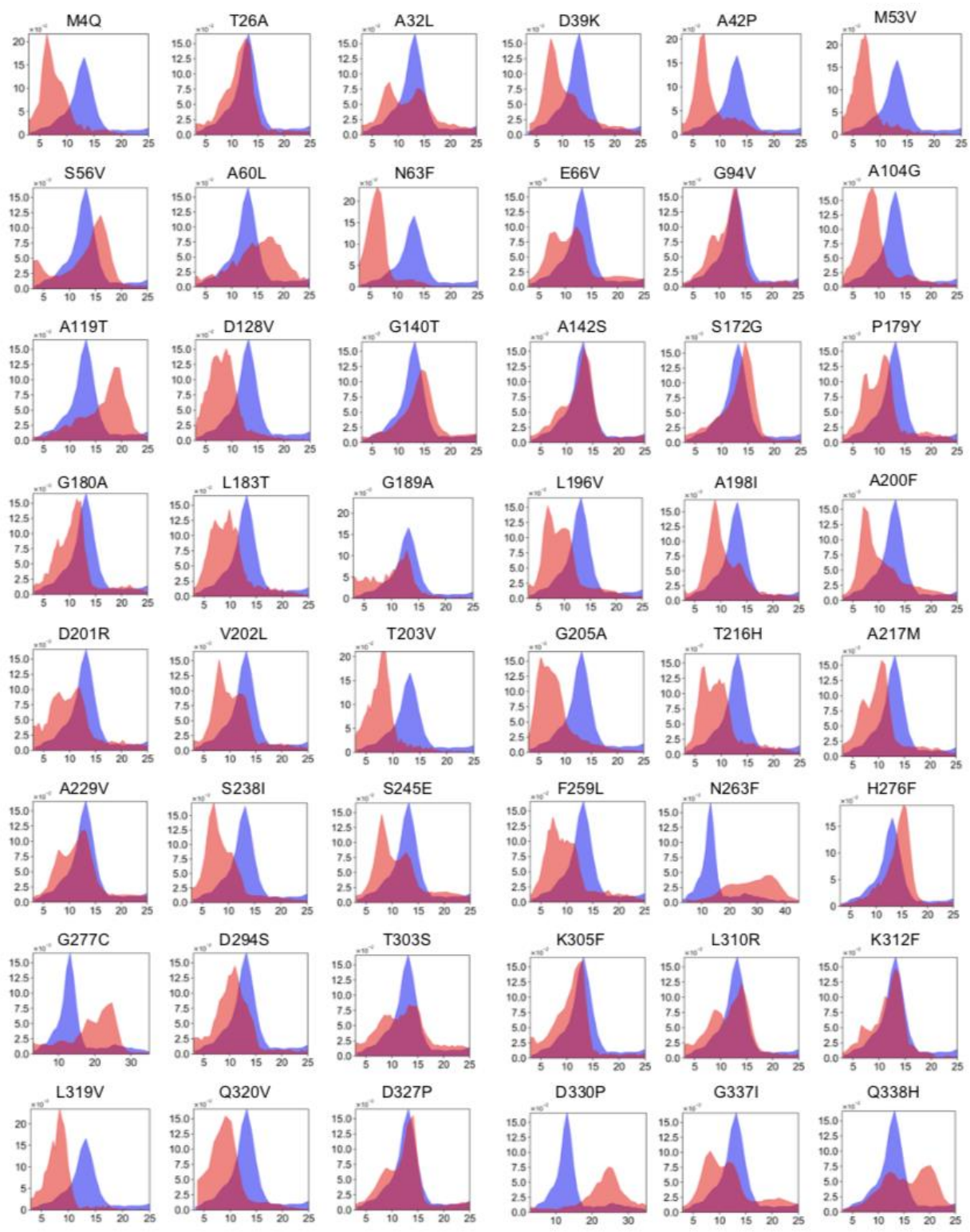

D330P

G3371

Q338H
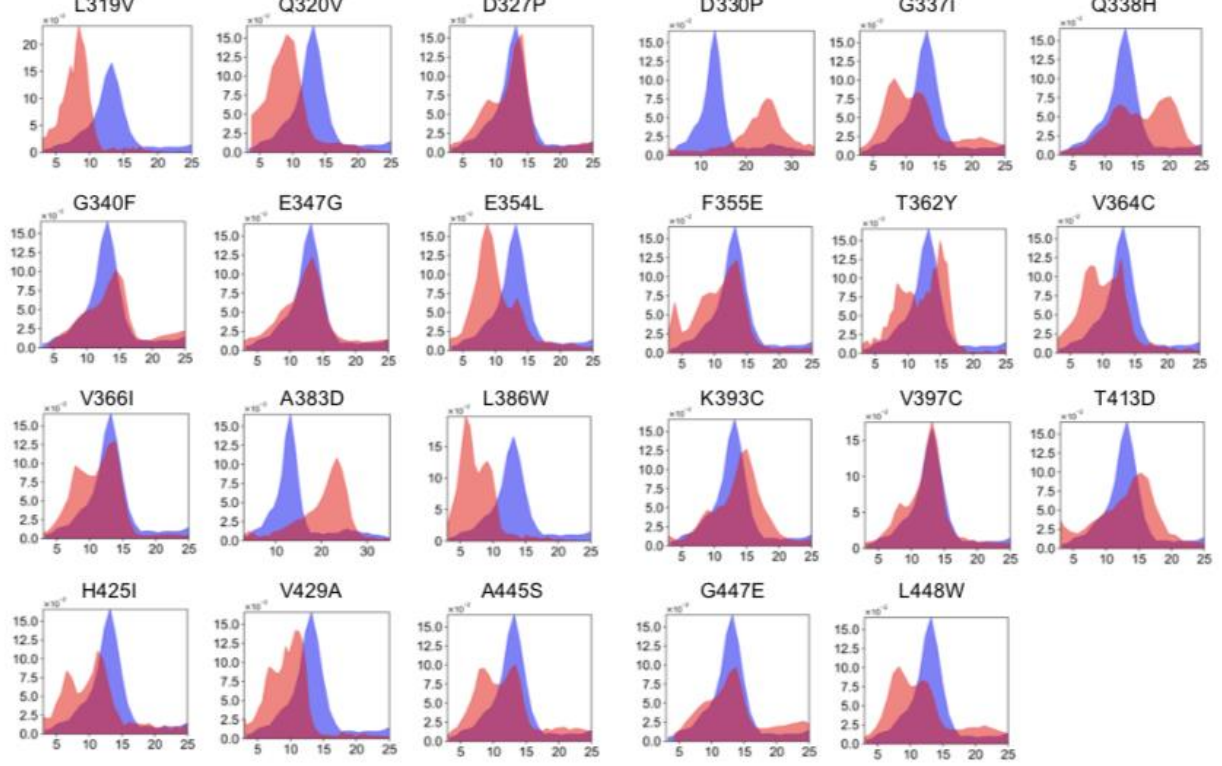

768 Supplementary Figure 7. Comparison of distribution of catalytic activities 
770 and population percentage of enzyme molecules (peaks 2 and 3). Red and blue bins

771 show the distribution of mutant APs and AP-wt, respectively, except for the highly

772 active mutants.

773 
a
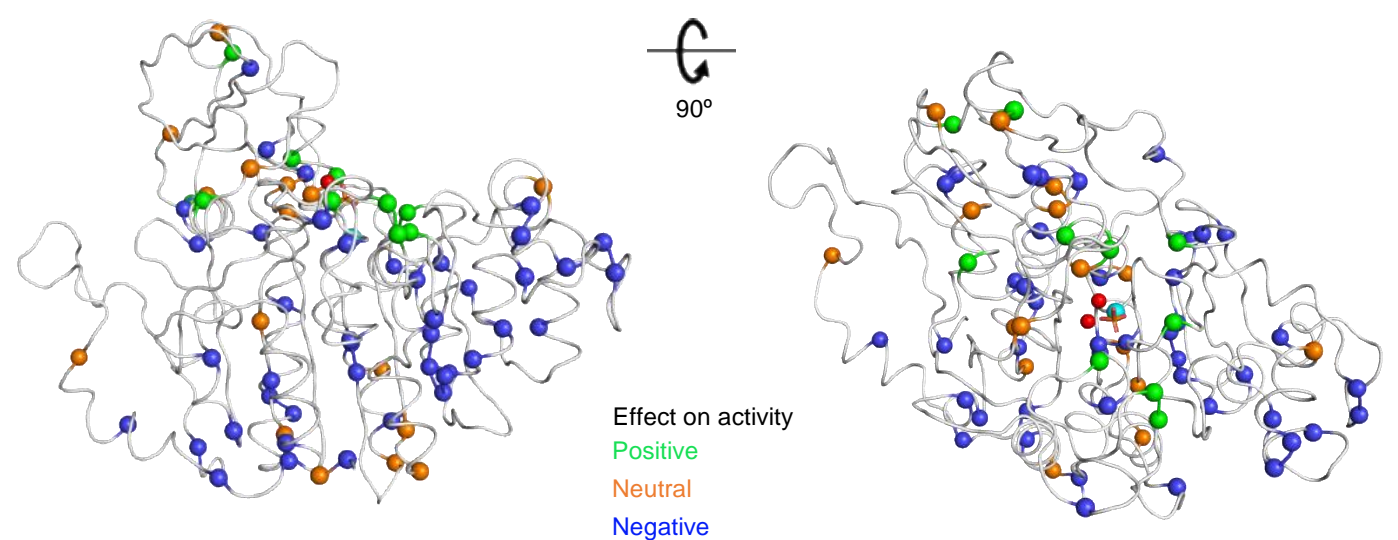

b
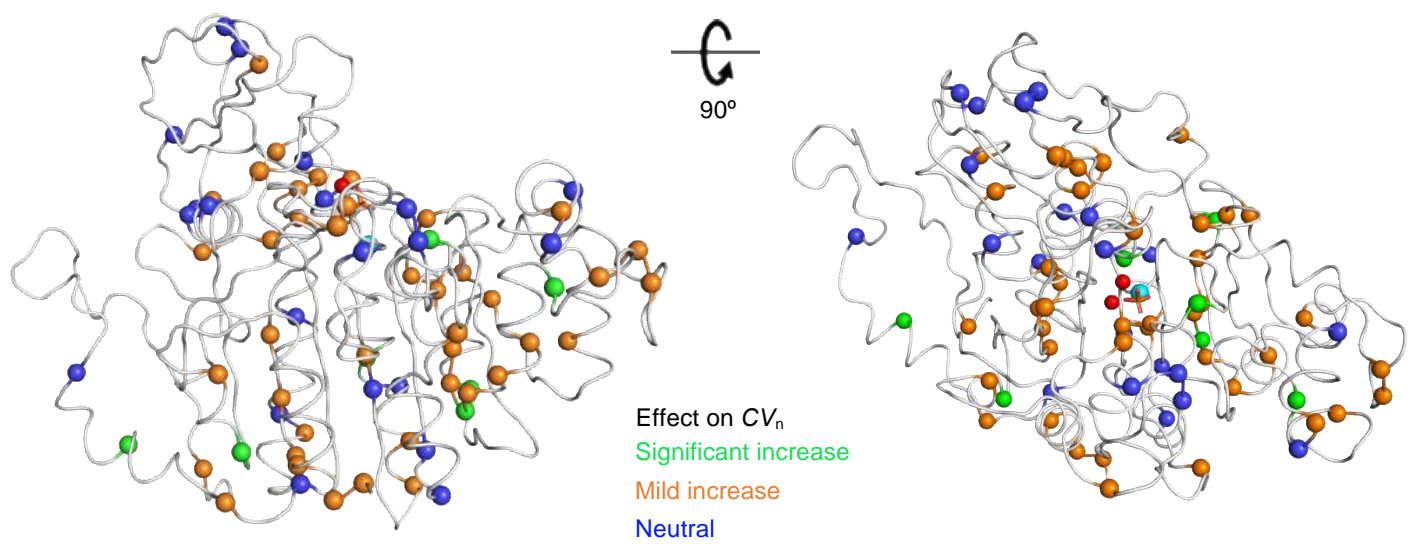

776 Supplementary Figure 8. Magnitude of effect of mutation on catalytic activity

777 (a) and functional heterogeneity (b) as shown on AP structure. Spheres

778 represent mutation sites and red and cyan spheres represent zinc and magnesium

779 ions, respectively. The color codes of mean catalytic activity $(M A)$ are separated as

780 follows; green (MA of mutant $>\mathrm{WT}+5 \times S D)$, orange (WT $-5 \times S D<$ mutant $\leq \mathrm{WT}$

$781+5 \times S D$ ), and blue (mutant $\leq \mathrm{WT}-5 \times S D$ ). Green, orange, and blue are

782 categorized as positive, neutral, and negative mutations, respectively. The color

783 codes for functional heterogeneity $\left(C V_{n}\right)$ are separated as follows; green $\left(C V_{n}\right.$ of

784 mutant $>\mathrm{WT}+5 \times S D)$, orange $(\mathrm{WT}+2 \times S D<$ mutant $\leq \mathrm{WT}+5 \times S D)$, and blue

785 (mutant $\leq \mathrm{WT}+2 \times S D$ ). Green, orange, and blue are categorized as significant

786 increase, mild increase, and neutral, respectively. 
a

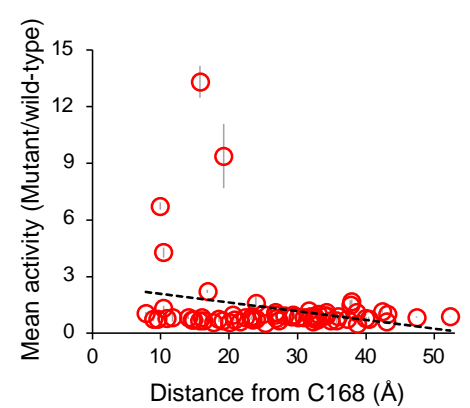

C

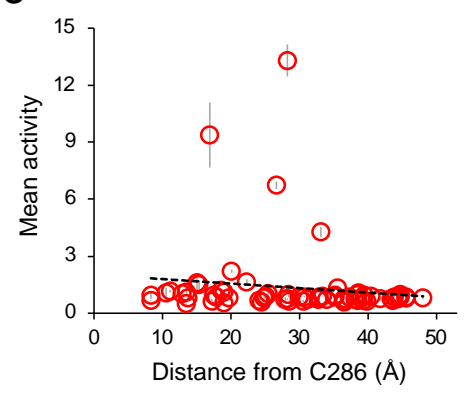

e

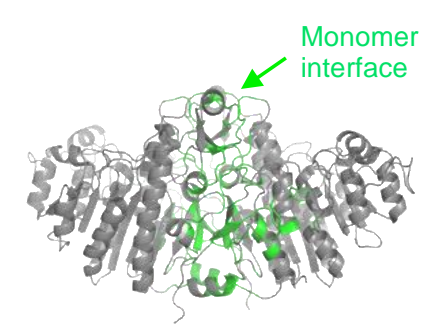

b

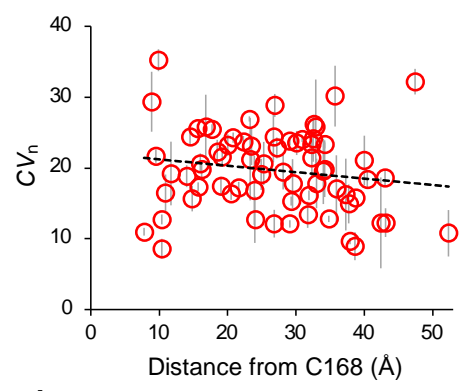

d

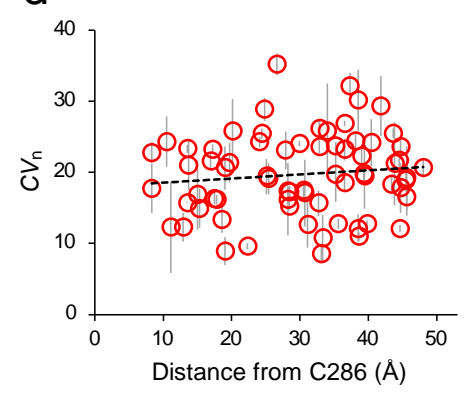

f

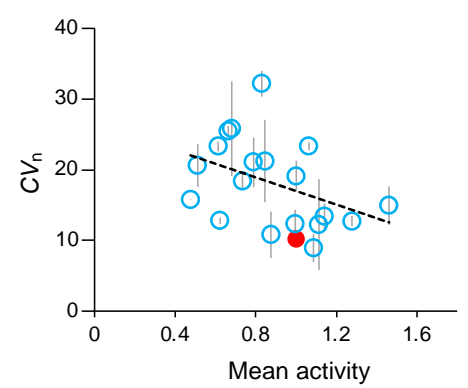

789 Supplementary Figure 9. Effects of mutations in catalytic activity and

790 functional heterogeneity on functionally important sites on AP. (a) and (b)

791 represent the dependence of mean catalytic activity $(M A)$ and functional

792 heterogeneity $\left(C V_{n}\right)$ on distance $(\AA)$ from $C 168$ (means $\left.\pm S D\right)$. The dotted lines show

793 linear fitting of the plots, and the slopes of $(a)$ and $(b)$ were $-0.05\left(R^{2}=0.06\right)$ and

$7940.09\left(R^{2}=0.03\right)$, respectively. $(\mathbf{c})$ and $(\mathbf{d})$ represent the dependence of AP activity

795 and $C V_{n}$ on distance $(\AA)$ from $C 286$ (means $\pm S D$ ). The slopes of $(\mathrm{c})$ and $(\mathrm{d})$ were -

$7960.02\left(R^{2}=0.02\right)$ and $0.06\left(R^{2}=0.01\right)$, respectively. (e) Sequences located at the

797 interface of the monomers are shown in green. (f) Correlation between enzyme

798 activity and functional heterogeneity involving mutations around the monomer

799 interface (mean $\pm S D$ ). The red circle represents AP-wt. The slope of the plot was -

$800 \quad 0.10\left(R^{2}=0.17\right)$. 